\title{
In-Band Full-duplex Dynamic Spectrum Sharing in Beyond 5G Networks
}

\author{
Sudip Biswas, Abhijeet Bishnu, Faheem A. Khan and Tharmalingam Ratnarajah
}

\begin{abstract}
Dynamic spectrum sharing (DSS) has recently attracted keen interest from regulatory bodies around the world as a key strategy to overcome spectrum scarcity in beyond 5G networks. In the USA, Federal Communications Commission (FCC) has adopted a three-tier DSS model for Citizens Broadband Radio Service (CBRS) band managed by a spectrum access system (SAS) to share incumbents' spectrum with commercial cellular broadband applications. In this article, we first present a survey of current DSS approaches and their limitations and then discuss the benefits of using in-band full-duplex (IBFD) techniques in DSS networks. To illustrate the improvement in the performance of priority access license (PAL) and general authorized access (GAA) users in an IBFD assisted CBRS, we consider a CBRS mobile broadband network (MBN) architecture comprising of an incumbent in the form of a MIMO radar system and an IBFD MIMO MBN, consisting of PAL and GAA users. In particular, we design joint beamformers at the $\mathrm{MBN}$, with constraints on transmit power at the MBN and detection probability of the radar system, and beamformers at the radar system to mitigate the interference from the radar system towards the cellular system. It is shown that the IBFD CBRS network architecture not only leads to improved performance of PAL and GAA users but does so while producing lesser interference towards the radar than state-of-theart half-duplex solutions. Finally, we present some open research challenges to invigorate research beyond 5G IBFD DSS networks.
\end{abstract}

\section{INTRODUCTION}

The recent deployment of 5G New Radio (NR) around the world has been categorized into two separate sets of frequency bands: < 7.225 GHz, known as frequency range 1 (FR1) and 24.25-52.6 GHz, known as FR2. While a much higher bandwidth is available in FR2 (millimeter-wave frequencies), it can only be used for short-range, high data rate applications. On the other hand, access to FR1 spectrum, is seriously hampered by archaic static spectrum allocation policies that permit the incumbent users exclusive access to its allocated bands while inhibiting others from accessing it [1].

Recently, there has been a major paradigm shift in the way radio spectrum is managed, which has garnered the interest of both industry and academia. To accentuate the acceptance of DSS, a global, not-for-profit, cross-sectoral organization, Dynamic Spectrum Alliance (DSA), comprising of Google, Amazon, Microsoft, Cisco, Broadcom, etc., has recently been formed to promote dynamic and unlicensed access to the spectrum, leading to more efficient spectrum utilization and to encourage innovation and economic growth. Several promising solutions such as cognitive radios (CRs), licensed shared access (LSA), TV white space, and spectrum access system (SAS) have been proposed to move towards flexible and dynamic spectrum allocation from the current rigid and static spectrum allocation [1], [2] (see Table 1). In the USA, Federal Communications Commission (FCC) has allowed SAS based spectrum sharing for secondary access through a three-tier shared access model consisting of incumbent access (IA), priority access license (PAL), and general authorized access (GAA) tiers in the $3.5 \mathrm{GHz}$ Citizens Broadband Radio Service (CBRS) band [3]. The incumbent users (IUs) operating in this band include federal users (military radars) and fixed satellite service earth stations who are protected from interference caused by PAL and GAA users through strict predefined guidelines. This forces the CBRS mobile broadband network (MBN) to either transmit in radar's white spaces or completely avoid any transmission inside predefined exclusive zones introduced by the National Telecommunications and Information Administration (NTIA) [4]. However, it is worth noting that a significant part of the US population resides in the exclusion zones, leading to very limited free spectrum opportunities being available for cellular operators utilizing the shared CBRS band in conjunction with incumbents. Hence, the present approach is very conservative in the sense that it not only puts the entire burden of maintaining an extremely high level of interference protection towards IUs on the MBN, but also does not inspire enough confidence for cellular operators to consider it as a business case. An alternative approach where the incumbents are designed with anticipation of spectrum sharing and cooperation with MBNs will lead to significantly higher utilization of the freed spectrum, boosting the case for widespread commercial deployment.

On a similar note, in-band full-duplex (IBFD) technology has recently shown promising results to effectively double the spectrum efficiency in communication systems [5]. In particular, an FD-DSS network can transmit and receive (sense) from (the transmission status of) other nodes at the same time and in the same frequency band. FD technology can also aid in combating several problems at the medium access control (MAC) layer, such as hidden terminals, congestion, and large delays. The performance of IBFD systems has long been constrained by high self-interference (SI) due to the proximity of the transmitter to its own receiver. However, recent advances in IBFD systems have shown that SI can be mitigated successfully to the extent that the true benefits of IBFD systems can be sustained [5]. These advantages have elevated IBFD to the pinnacle of beyond $5 \mathrm{G}$ communications, which is evident from the fact that several start-ups on FD have been recently backed by technological giants such as Verizon, Cisco, TMobile, and Intel. Accordingly, in the alternative CBRS dynamic spectrum sharing (DSS) approach, suggested above, spectrum efficiency can be further increased for the MBN by transmitting in IBFD mode, leading to enhanced quality of service (QoS) for the PAL/GAA users. 


\begin{tabular}{|c|c|c|c|c|c|}
\hline $\begin{array}{c}\text { Spectrum } \\
\text { Sharing Approach }\end{array}$ & $\begin{array}{l}\text { Frequency } \\
\text { Band }\end{array}$ & $\begin{array}{l}\text { Sharing } \\
\text { Structure }\end{array}$ & $\begin{array}{l}\text { Management } \\
\text { Blocks }\end{array}$ & Applications & Limitations \\
\hline $\begin{array}{l}\text { Cognitive } \\
\text { Radio (CR) }\end{array}$ & $\begin{array}{l}\text { TV White } \\
\text { Space } \\
\text { 470-790 } \\
\text { MHz (UK) }\end{array}$ & Interweave & $\begin{array}{c}\text { Spectrum } \\
\text { sensing/Database } \\
\text { assisted }\end{array}$ & $\begin{array}{c}\text { Rural } \\
\text { broadband }\end{array}$ & $\begin{array}{c}\text { No QoS } \\
\text { guarantees for } \\
\text { secondary users }\end{array}$ \\
\hline $\begin{array}{c}\text { Licensed } \\
\text { Shared Access } \\
\text { (LSA) }\end{array}$ & $\begin{array}{l}2.3-2.4 \\
\mathrm{GHz} \\
\text { Europe }\end{array}$ & $\begin{array}{c}\text { Two-tier } \\
\text { sharing } \\
\text { (incumbent and } \\
\text { licensee) }\end{array}$ & $\begin{array}{c}\text { LSA } \\
\text { Repository, LSA } \\
\text { Controller }\end{array}$ & $\begin{array}{r}\text { Mobile } \\
\text { Broadband }\end{array}$ & $\begin{array}{c}\text { Target } \\
\text { stakeholders } \\
\text { (MNOs) still } \\
\text { focused on } \\
\text { dedicated licensed } \\
\text { spectrum }\end{array}$ \\
\hline $\begin{array}{c}\text { Citizens } \\
\text { Broadband Radio } \\
\text { Service- Spectrum } \\
\text { Access System } \\
\text { (CBRS-SAS) }\end{array}$ & $\begin{array}{c}3.55-3.7 \\
\text { GHz USA }\end{array}$ & $\begin{array}{c}\text { Three-tier } \\
\text { sharing model } \\
\text { involving IA, } \\
\text { PAL and GAA } \\
\text { users }\end{array}$ & $\begin{array}{c}\text { Spectrum } \\
\text { Access System }\end{array}$ & $\begin{array}{r}\text { Mobile } \\
\text { Broadband }\end{array}$ & $\begin{array}{c}\text { No QoS } \\
\text { guarantees for GAA } \\
\text { users }\end{array}$ \\
\hline
\end{tabular}

Table 1: Comparison of current dynamic spectrum sharing approaches

Based on the above, in this article, we aim to tackle the topics of spectrum paucity and its under-utilization by first surveying the current DSS scenario and then describing a new IBFD CBRS network architecture that leads to i) more efficient utilization of the shared CBRS band, whereby the PAL/GAA users do not have to vacate the spectrum whenever the IU is using it and ii) lesser interference from MBN towards the radar than state-of-the art half-duplex (HD) solutions (radios that either transmit or receive at any particular time or frequency). We show that DSS in beyond $5 \mathrm{G}$ networks will have to seriously consider the adoption of IBFD technology to further increase the utilization of shared spectrum.

The article is organized as follows. Section II first presents the current dynamic spectrum sharing approaches and their limitations. It then describes IBFD based DSS approach by elaborating IBFD communication principles, benefits, and current state-of-the-art SI mitigation techniques. Section III details an IBFD CBRS network architecture and elaborates on the achieved results and improvement in performance. Section IV outlines the future research challenges in DSS networks. Finally, Section V summarizes the article.

\section{DYNAMIC SPECTRUM SHARING FOR BEYOND 5G NETWORKS}

\section{A. Current DSS Approaches and their Limitations}

Among the various DSS approaches, CR was proposed as a promising spectrum sharing approach that allowed unlicensed users to transmit in the spectrum band owned by the primary user, by detecting spectrum holes at any given time/frequency/location while ensuring no harmful interference to primary users. The existing concerns over technical capabilities to ensure successful spectrum sensing all the time and risk of interference to primary users have restricted the deployment of CR. FCC mandated database-driven approach to prevent harmful interference to the primary users alleviated these concerns to some extent. However, it was still insufficient to deploy CR for commercial applications. Another drawback of the traditional CR approach was the lack of QoS guarantee to the secondary users, as they were required to vacate the spectrum as soon as the primary user started its own transmission. This further highlighted the limitations of this approach [1],[6].

More recently, another important development towards DSS has risen: the co-existence of commercial cellular providers and federal incumbents (e.g., military radars), commonly referred to as communication-radar (ComRad) coexistence [4],[7]. Two approaches, in particular, have received significant attention: licensed shared Access (LSA) and spectrum access system (SAS) in the CBRS band of 3.55$3.7 \mathrm{GHz}$, also known as LTE band 48. LSA was introduced in Europe in 2.3-2.4 GHz, in which additional licensed users, referred to as licensees, could access the spectrum band of the incumbents. In the USA, an alternative DSS scheme is approved by FCC in the CBRS band for spectrum sharing via a mix of licensed and unlicensed spectrum. Fig. 1 shows an overview of the spectrum access of CBRS within three levels of services based on SAS. However, the primary drawback of SAS is that it is also a conservative approach, where the GAA users which were supposed to be the main beneficiaries of SAS have been placed at the bottom of the pyramid, whereby they either fall in the NTIA defined exclusion zones or suffer from severe interference from the higher tiers. Such a cautious approach has shown limited potential that has hindered its commercial success. Further, just transmitting in radar's white spaces is not contemplated profitable enough for mobile operators.

Accordingly, accommodating wireless communications within the radar spectrum involves a game-changing paradigm, which cannot be addressed with existing spectrum sharing approaches [9]. In typical spectrum sharing scenarios, both licensed and unlicensed applications involve communication links. In ComRad spectrum sharing, however, the links involving the incumbent include a radar application that essentially has 


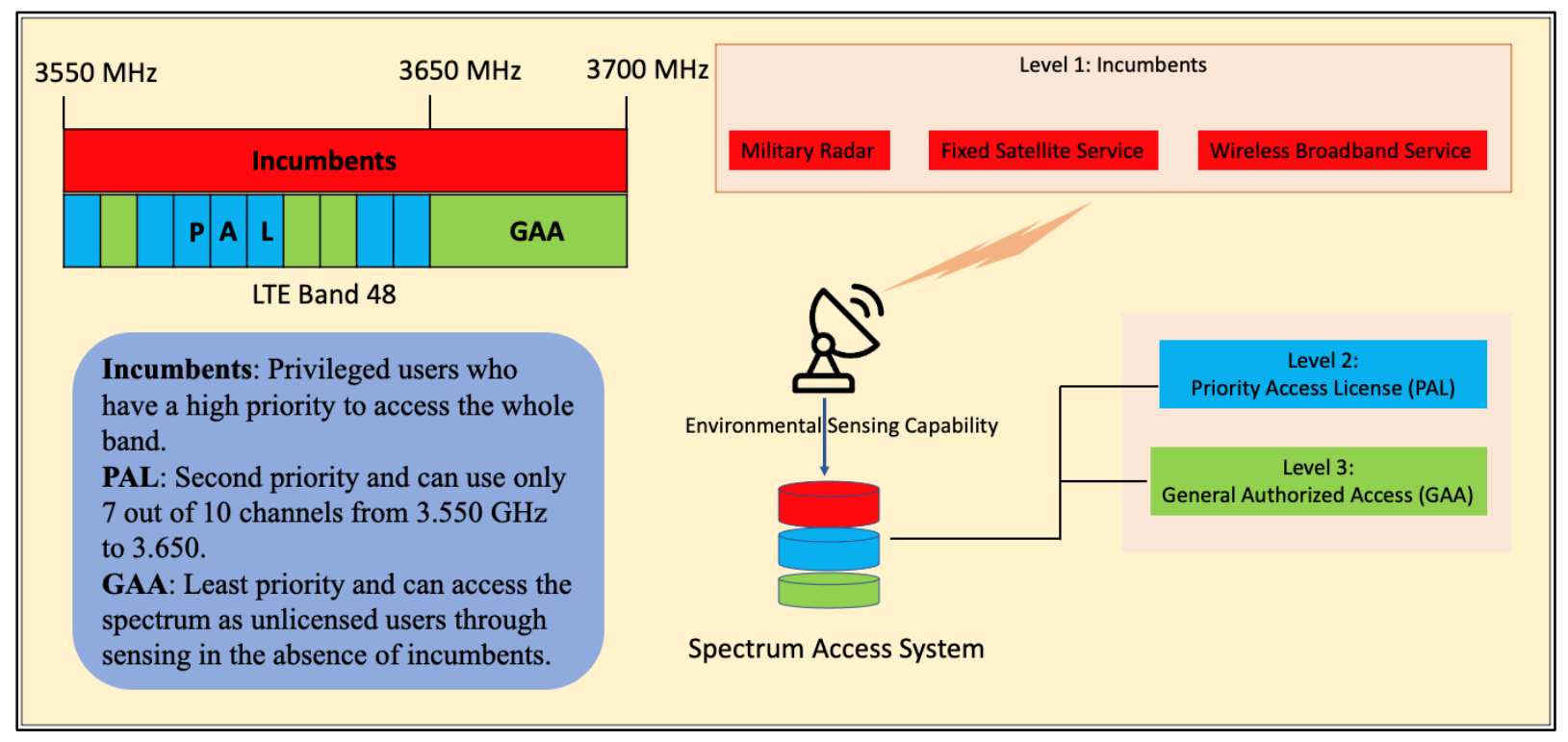

Figure 1: Three tier CBRS based on SAS as approved by FCC.

distinctive function and vital performance metrics, due to which the joint design of the two systems becomes challenging. Furthermore, the radar systems often involve critical applications such as aviation traffic control and defense applications which necessitate extreme reliability, i.e., errorfree operation $100 \%$ of the time. In recent years, the interference in radar bands is increasing as the usable spectrum has been allocated to the latest communication technologies. This has highlighted the need to protect critical radar operations in applications such as aeronautical and weather radar, radar for airport traffic management, etc., operated by the military and governmental organizations. Accordingly, existing spectrum sharing solutions based on generic interference constraints are not applicable, as they fail to address particular performance constraints of radar systems, both in terms of metrics and in terms of the level of reliability requirements [7].

\section{B. IBFD for Beyond $5 G$ Networks}

DSS techniques have recently led to more efficient utilization of spectrum which can be further improved by deploying IBFD communication technology. It has recently been demonstrated through advanced signal processing techniques that simultaneous transmit and receive communication through IBFD radio is indeed feasible. The SI cancellation can primarily be classified under passive and active domains. Passive cancellation takes place before the transmitted signal enters the receiving antenna of the IBFD radio. Passive cancellation is performed by utilizing different antenna and signal propagation characteristics, such as separation among antennas, shielding of antennas, and effects of antenna polarization. Active cancellation mainly constitutes cancellation in the analog and digital domain [5].

Analog cancellation is used to mitigate the SI before it enters the analog-to-digital converter (ADC). One particular example of analog cancellation is described in [10], where the attenuation and delay of the SI channel is iteratively estimated and then a negative signal is generated through a balun transformer and is combined with its received signal to cancel the SI before ADC. Other techniques include sequence-based methods, combinations of time-domain algorithms, and photonic integrated circuits. However, in reality, SI cancellation is non-ideal in nature and is severely limited by hardware impairments caused by nonlinearities of the power amplifiers, I/Q imbalance and phase noise, imperfect channel estimation, the limited resolution of $\mathrm{ADC} /$ digital to analog converter etc. As a result, residual SI (RSI) is the primary predicament for IBFD that is currently plaguing the promised $2 \mathrm{X}$ capacity improvement over corresponding HD systems [10]. This RSI can be mitigated digitally by designing optimal digital beamformers, i.e., precoder and receiver matrices, which is the final phase of SI cancellation. In particular, each antenna, analog, and digital cancellation theoretically account for around $20 \mathrm{~dB}, 50 \mathrm{~dB}$, and 30-35 dB cancellation, respectively and often in practice, they are all implemented together to achieve the desired level of SI cancellation.

However, the SI cancellation techniques which are available off-the-shelf are mostly for narrow-band systems and may not be applicable for radars, which mostly operate in wide-band channels along with carrier aggregation. To this end, optical domain-based novel canceller architecture has been proposed for SI cancellation in wideband channels [13] in beyond 5G systems. This innovative optical domain-based canceller provides $20 \mathrm{~dB}$ of RF cancellation, achieved over 1 $\mathrm{GHz}$ bandwidth centered at $2.5 \mathrm{GHz}$ with 20 utilized taps.

\section{IBFD Based DSS for Beyond $5 G$ Networks}

The limitations imposed on current DSS approaches have resulted in a significant technology revolution through the advent of IBFD DSS networks, where secondary (PAL/GAA) users can benefit from: i) simultaneous sensing and transmission (SST) and ii) simultaneous transmission and reception (STR) [4]. By utilizing proper SI mitigation technique at the IBFD spectrum sharing node, both sensing efficiencies, as well as secondary throughput can be maximized. A 


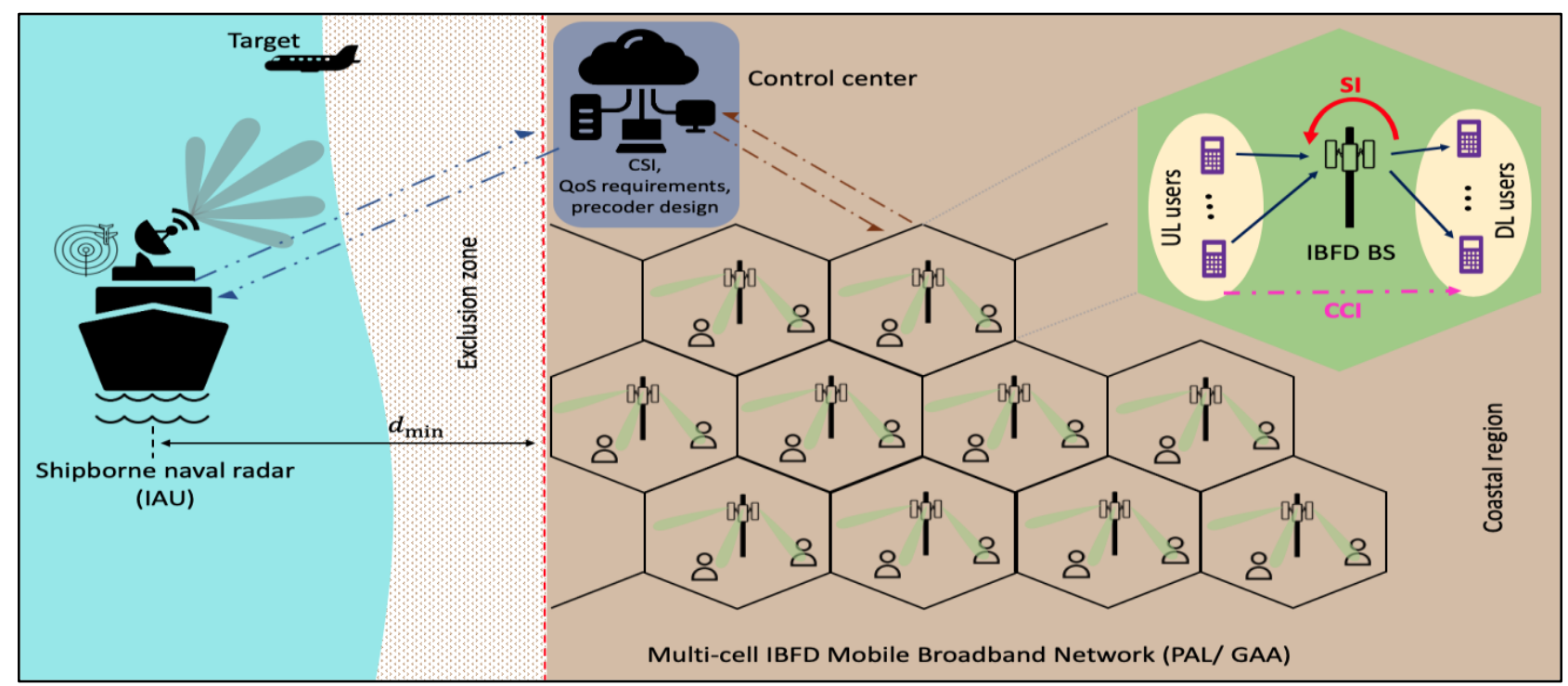

Figure 2: IBFD CBRS Network Architecture

comparison of spectrum sensing and exploitation techniques of IBFD spectrum sharing with respect to HD spectrum sharing is given below:

1. Secondary throughput: IBFD spectrum sharing networks (SSN) ensures higher secondary throughput in comparison to HD SSN as unlike in HD spectrum sharing, data transmission is not interrupted by the sensing operation.

2. Improved sensing and interference mitigation: In HD SSN, the users in the secondary network cannot detect incumbent/radar activity during data transmissions, which may result in interference towards the incumbent network/ radar. However, in IBFD SSN, the incumbent/radar's activity can be constantly monitored, which not only improves the sensing performance compared to HD SSN but also minimizes the interference towards the incumbent network/ radar.

3. Collision avoidance efficiency: Collision avoidance among the users of the secondary network is another advantage of IBFD spectrum sharing over HD spectrum sharing. Unlike in IBFD SSN, the collision duration during transmission in HD SSN is usually large as collision detection is usually intermittent during transmission.

4. Energy efficiency: IBFD SSN can be more energy-efficient than its HD counterparts as energy can be harvested by users in the secondary network without requiring to discontinue spectrum sensing and data transmission activity.

5. Security through jamming: Data transmission security is a critical aspect of SSN. In IBFD SSNs, the users of the secondary network can simultaneously receive data and transmit jamming signals to improve the secrecy performance of the network.

\section{IBFD CBRS NETWORK ARCHITECTURE}

The IBFD CBRS network architecture is shown in Fig. 2. In particular, here we present a case study that illustrates the beamforming design solution, where PAL/GAA users get higher priority than in conventional CBRS, while still achieving the desired performance (detection probability for the case of a radar) for the IU. This is achieved through the application of i) a two-stage beamforming design algorithm, where both IU and PAL/ GAA actively cancel each other's interference and ii) IBFD radios at the PAL/GAA users. Unlike conventional CBRS, where the PAL/GAA users are required to vacate the spectrum as soon as IU users are sensed, the proposed approach ensures that the PAL/GAA users can always access the spectrum with assured quality of experience (QoE). In particular, the modified CBRS inspires greater confidence to become a business case for mobile broadband networks (MBNs) in the PAL/GAA regime.

\section{A. Simulation Framework and Network Parameters}

A remote coastal area devoid of any cellular coverage is considered. In particular, a cooperative spectrum sharing framework, where an MBN co-exists with a maritime MIMO radar (e.g., US navy's AN/SPN-43C air traffic control radar operating in the $3.5 \mathrm{GHz}$ FR1 band). The MBN consists of multiple small-cells deployed under the 3GPP NR specifications [12], where each cell has a radius of $50 \mathrm{~m}$ and consists of a MIMO base-station (BS) that operates in IBFD mode and serves multiple HD MIMO users in both uplink (UL) and downlink (DL). While the MIMO radar, located in the ocean at a distance $d_{\min }=400 \mathrm{~m}$ from the coast is the IU, the operators/ users in the MBN located in the coastal region can be considered as the PAL/ GAA. A MIMO radar [4], [8] is considered because it achieves higher degrees of freedom than traditional phased-array radars. This is due to its enlarged virtual array aperture owing to its orthogonal waveforms. Adhering to the guidelines of CBRS, an exclusion zone is maintained between the IU and the PAL/ GAA. Apart from the RSI due to IBFD operation, co-channel interference (CCI) from UL users to DL users, and inter-cell interference (ICI) are also considered. The CCI in the network can be handled through smart channel assignment using scheduling techniques such as game theory before the precoder/ receiver design. The thermal noise density is set at $-174 \mathrm{dBm} / \mathrm{Hz}$ and the noise figures at the IBFD BSs and UEs are set at $13 \mathrm{~dB}$ and $9 \mathrm{~dB}$ respectively [11]. 


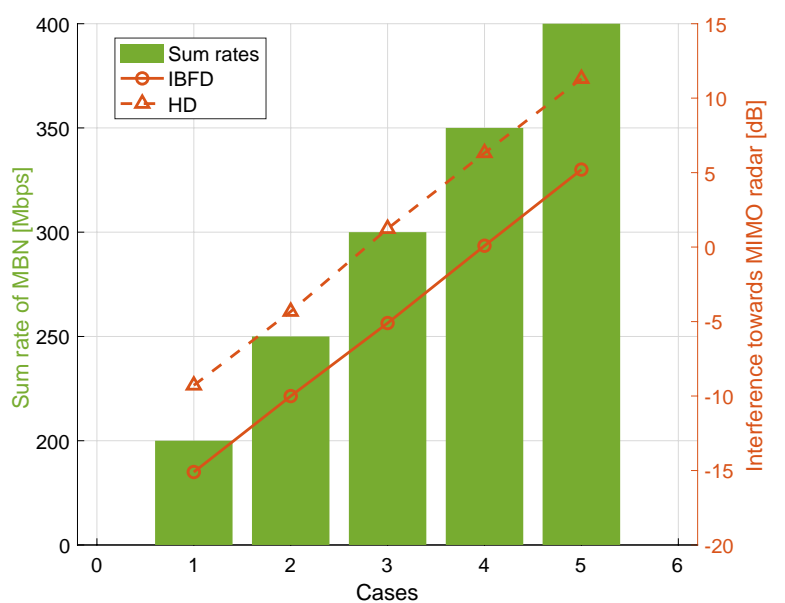

Figure 3: Interference from MBN towards IU for different QoS

The path loss exponents for line-of-sight (LOS) and non-lineof-sight (NLOS) are set as 2.0 and 3.1, respectively, while the standard deviations of the shadow fading for LOS and NLOS are set at $2.9 \mathrm{~dB}$ and $8.1 \mathrm{~dB}$, respectively. Further, the SI channel is modeled according to the Rician model in [11].

Now utilizing the interference channels shared by the MIMO radar, we focus on the design of a modified CBRS control center, whereby the CBRS controller warrants that the MBN involving the PAL/ GAA users always have access to specific quality of service (QoS), for which the combined interference towards the radar from the MBN is constrained. Accordingly, a two-step beamforming structure, where passive beamforming is implemented to mitigate the interference from the MIMO radar towards MBN. In particular, we design the precoding matrices at the MIMO radar via a subspace method. Next, active beamforming matrices at the MBN are designed to maximize the MIMO radar's detection probability $\left(P_{d}\right)$ with constraints on QoS of the PAL/ GAA users in individual cells and transmit powers at the UL PAL/ GAA users and the IBFD BSs.

The simulations are performed for a carrier frequency of $3.55 \mathrm{GHz}$ and a bandwidth of $10 \mathrm{MHz}$, in accordance with the specification of radar spectra available for sharing in [14]. $15 \mathrm{KHz}$ subcarrier spacing is used with an FFT size of 1024 subcarrier and 864 data subcarriers. 72 resource blocks are used for data transmission. Unless otherwise stated, the following configurations are considered for the radar: number of antennas $=8$, probability of false alarm $=10^{-5}$, velocity of target 782 knots, and distance of the target from radar $=300 \mathrm{~m}$. Similarly, for the MBN, $G=3$, RSI cancellation strength is set at $-70 \mathrm{~dB}$ and $\mathrm{CCI}$ attenuation factor is set at $0.5, \mathrm{QoS}=300 \mathrm{Mbps}$.

\section{B. Simulation Results}

We begin by showing the performance (in terms of guaranteed QoS for the MBN) of the proposed spectrum sharing framework and the corresponding advantages of using IBFD radios over HD in Fig. 3. The number of considered events are shown in the $x$-axis, the QoS requirements (in terms of sum rate in Mbps) for the MBN is shown in the left $y$-axis and the equivalent minimized interference towards the radar is presented in the right $y$-axis. In particular, we consider five cases, with each case denoting a QoS requirement as labeled on

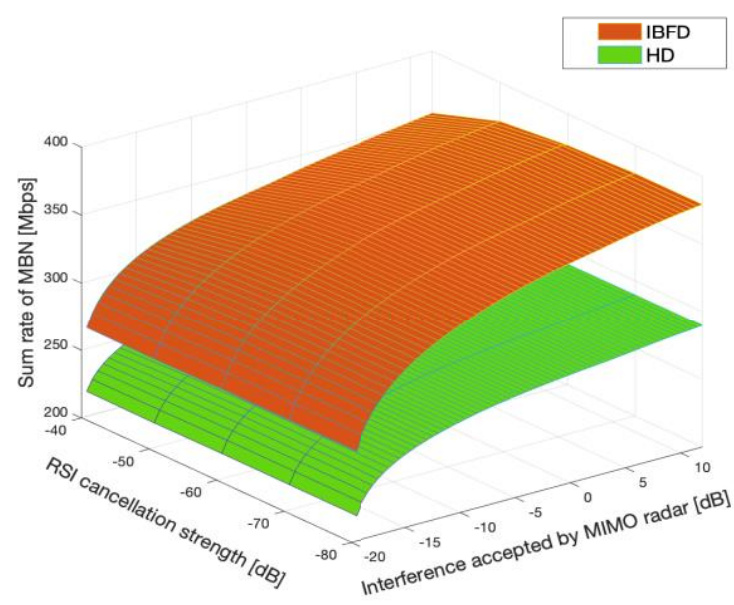

Figure 4: Sum rate of the MBN vs RSI cancellation strength vs Interference accepted by MIMO radar

the left $y$-axis. It can be seen from the figure that to maintain a specific sum rate at the MBN, interference exposure from MBN towards MIMO radar is higher for HD than IBFD. For instance, for the $3^{\text {rd }}$ Case (i.e., for sum rate $=300 \mathrm{Mbps}$ ), the collective interference at MIMO radar from the $\mathrm{HD} \mathrm{MBN}$ is $\approx 1 \mathrm{~dB}$, while it is only $\approx-5 \mathrm{~dB}$ for the IBFD case. The level of interference further rises when the requirements of $\mathrm{QoS}$ at the MBN increases. This can be explained as: the IBFD MBN utilizes the full spectral resources and hence to attain a specific sum rate, less transmit power is required by the IBFD MBN than its HD counterpart. Accordingly, the MBN with IBFD radios produces minimal interference and is particularly advantageous for SS scenarios. However, any such gains are subject to appropriate RSI attenuation, which we show in the following example.

In Fig. 4 we show the sum-rate of the IBFD MBN as a function of varying RSI cancellation strengths and the interference exposure accepted by the MIMO radar to achieve a particular detection probability. This study assumes that prior antenna/ analog cancellation has been performed and accordingly, centers around RSI cancellation in the digital domain. Nevertheless, existing analog domain cancellation methods can be added as an antecedent block to our design to realize an all-inclusive architecture. It can be seen from the figure that increasing the interference exposure towards the MIMO radar allows an improved sum rate in the MBN. In order to ensure a high detection probability of the radar, the proposed design ensures that after a certain threshold the sum-rate no longer increases linearly and gradually saturates. It can be seen that an interference exposure level of approximately $-5 \mathrm{~dB}$ is adequate to warrant a sizeable sum rate for the IBFD MBN. Furthermore, it can be observed that the IBFD operation increases the performance of the MBN by approximately $60 \%$ over conventional HD operation. This gain is dependent on the cancellation capability of RSI of the IBFD system, evident from the figure. In particular, a higher RSI cancellation level ensures a higher gap in performance between IBFD and HD operations.

In Fig. 5, we quantify the performance of the MIMO radar in terms of its detection probability of a target in the far-field as a function of transmit power and the number of antennas. It can be seen that for a fixed probability of false alarm, extra power 


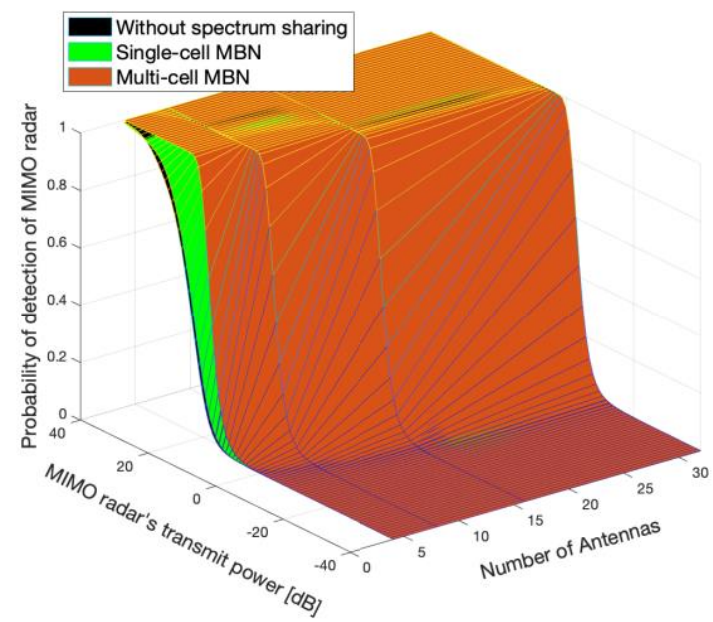

Figure 5: Probability of detection of the MIMO radar

is required by the MIMO radar to achieve a specific probability of detection, when a comparison is made with the scenario without spectrum sharing. However, it must be noted that the added power is not significant when the gains achieved by the MBN are put into consideration. In particular, $2-3 \mathrm{~dB}$ extra power is required to achieve a detection probability of 0.9 at the radar. Further, this additional power can be easily achieved by increasing the antenna number on the radar. This can be explained by the fact that whilst the antenna number at the MBN (BSs and users) is fixed, the radar's degrees of freedom increases when the antenna number is increased, which in turn enhances its reliability to detect a target and simultaneously null interference towards the MBN. This demonstrates that antenna arrays with large dimensions can be used at the radar to enable DSS without degrading the radar's performance.

In summary, an IBFD MBN can realize an aggregate sum rate of around $300 \mathrm{Mbps}$ for an RSI cancellation of $-70 \mathrm{~dB}$ while sharing the spectrum of MIMO radar. Further, MIMO radar requires 2 to $4 \mathrm{~dB}$ additional power to enable spectrum sharing while sustaining a detection probability of approximately 0.9 , based on the number of antennas (the maximum number of antennas supported by $5 \mathrm{G} \mathrm{NR} \mathrm{FR1} \mathrm{is} 32$ ). It is worth noting that this is a reasonable performance especially considering that sharing of the spectrum is supplemented with varied benefits that are conjointly established between incumbents (federal agencies) and MBN (cellular operators) in advance.

\section{Acquisition of CSI, implementation, and complexity of the CBRS controller}

The CBRS controller acquires the network's CSI with the capacity to exchange the CSI between the radar and the MBN. Ensuring a centralized methodology, the CBRS controller collects all the CSI data, and the QoS requirements from the MBN computes all the required transmit beamforming matrices and then dispenses them to the respective IBFD BSs and PAL/ GAA users. While the detector statistics of the waveforms of the radar projected in the null-space of the MBN are derived through a generalized likelihood ratio test for target detection, the monotonically increasing relationship between detection probability and its non-centrality parameter is exploited to resolve the detection probability in terms of interference exposure on the radar. The resulting non-convex active beamforming optimization problem is converted into a secondorder cone programming problem (SOCP) with definite convergence, whereby in each iteration a convex SOCP subproblem is efficiently solved by standard SOCP solvers using interior-point methods with polynomial complexity.

\section{IBFD DSS: FUTURE RESEARCH CHALLENGES}

While IBFD renders many conventional problems in spectrum sharing less relevant, it uncovers entirely new problems that require extensive research. A few such open problems are discussed below.

\section{A. IBFD Dual-Function ComRad BS}

Joint design approaches are indispensable for achieving the ComRad coexistence. Dual-functional systems with the ability to perform remote sensing of objects and transmit useful information via classical communications simultaneously will act as an essential enabler for future wireless radios. The dualfunctional ComRad system (DFCR) presents key advantages in that a dual-functional design can drastically cut the hardware complexity, cost, and volume of a wide range of electronic equipment used for both communications and radar applications. Also, the joint radar and communication signaling can be designed to improve the real-time performance for both applications [4]. While appealing as a theoretic concept, this still faces a number of critical challenges: (i) the fundamental limits of DFCR operation are widely unknown and necessitate a new theoretical framework that jointly addresses both systems. (ii) existing signaling for communications (radar) is not fundamentally tailored for radar (communications) functionality. This necessitates fundamental waveform design for DFCR. (iii) classification and recognition of the interfering signals for DFCR systems is still an open problem, while spectrum occupancy models are mathematically challenging. Machine learning methods that provide enabling solutions without the need for mathematically tractable models are essential.

\section{B. Secure and Energy Efficient IBFD SSN}

FD enabled GAA/PAL users can simultaneously receive the desired signals as well as transmit jamming signals towards eavesdroppers. Nevertheless, there exists a trade-off between the GAA/PAL user throughput and the secrecy performance, which requires further investigation through transceiver design and resource allocation [15]. Furthermore, for the case of ComRad, the licensed link involves a radar, often involving critical applications such as aviation traffic control, and defense applications which necessitate extreme levels of security. Based on the scenario of co-existence such as military-to-communication sharing or military-to-military sharing, reliability, and the level of protection for the radar must be further explored. Moreover, FD enabled GAA/PAL users can simultaneously harvest the energy while transmitting to intended users. Unprecedented gains are to be harnessed by exploiting radar interference constructively through energy harvesting in communication systems, which is unexplored to date.

\section{Adaptive MAC Layer}


The advantages of IBFD are significantly affected by i) spatial frequency reuse and ii) asynchronous contention. Hence, efficient MAC layer protocols are required to translate the FD gain from the physical layer to the network layer. One promising solution is to enterprise an adaptive MAC protocol, that enables the node to operate on either IBFD or HD based on environmental interference with some objective functions. In addition, an efficient MAC protocol is also necessary to address the fairness of non-uniform users with unbalanced traffic distribution in the IBFD wireless network.

\section{SUMMARY}

A promising method for tackling the issue of spectrum scarcity in future wireless networks is DSS and IBFD. This article has discussed some major features of IBFD radios and the corresponding implications of it being used in conjunction with DSS and ComRad networks. An IBFD CBRS network architecture was presented to alleviate the shortcomings of existing CBRS systems. In particular, we proposed a modified CBRS controller architecture, involving a two-step beamformer design with the objective of i) mitigating the interference from the MIMO radar towards $\mathrm{MBN}$, and ii) maximizing the detection probability of the MIMO radar subject to QoS constraints of the PAL/ GAA users in each cell of the MBN. Numerical results demonstrated the efficacy of the IBFD design albeit certain trade-offs in radar/ MBN transmit power, number of antennas, SI cancellation strength, detection probability of the radar, and QoS requirements for the PAL/ GAA users. The interference generated by the IBFD CBRS framework towards the radar was found to be less than baseline HD CBRS systems, while also providing the PAL/ GAA users with enhanced throughput. Lastly, a few noteworthy open research problems with regards to the operation of IBFD for DSS were reviewed with the aim of fast-tracking future research activities in this domain.

\section{ACKNOWLEDGMENT}

The work of S. Biswas was supported by the Science and Engineering Research Board (SERB), Government of India, under Grant SRG/2020/001145. The work of T. Ratnarajah and A. Bishnu was supported by the UK Engineering and Physical Sciences Research Council (EPSRC) under Grant $\mathrm{EP} / \mathrm{P} 009549 / 1$.

\section{REFERENCES}

[1] C. B. Papadias, T. Ratnarajah, and D. T. M. Slock, "Spectrum Sharing: The Next Frontier in wireless Networks," Wiley-IEEE Press, Apr. 2020.

[2] G. K. Papageorgiou et al., "Advanced Dynamic Spectrum 5G Mobile Networks Employing Licensed Shared Access," IEEE Commun. Mag., vol. 58, no. 7, pp. 21-27, July 2020.

[3] Federal Communications Commission. FCC Proposes Innovative Small Cell Use in $3.5 \mathrm{GHz}$ Band. Accessed: Dec. 2012. [Online]. Available: https://www.fcc.gov/document/fcc-proposes-inovative-small-cell-use35-ghz-band.

[4] S. Biswas et al., "Design and Analysis of FD MIMO Cellular Systems in Coexistence With MIMO Radar," IEEE Trans. Wireless Commun., vol. 19, no. 7, pp. 4727-4743, July 2020.

[5] A. Sabharwal, et al., "In-Band Full-Duplex Wireless: Challenges and Opportunities," IEEE J. Sel. Areas Commun., vol. 32, no. 9, pp. 16371652, Sept. 2014.
[6] F. A. Khan et al., "Interference Driven Linear Precoding in Multiuser MISO Downlink Cognitive Radio Network," IEEE Trans. Veh. Tech., vol. 61, pp. 2531-2543, June 2012.

[7] S. Biswas et al., "Coexistence of MIMO Radar and FD MIMO Cellular Systems with QoS Considerations," IEEE Trans. Wireless Commun., vol. 17, no. 11, pp. 7281-7294, Nov. 2018.

[8] J. Li and P. Stoica, "MIMO Radar with Colocated Antennas," IEEE Sig. Process. Mag., vol. 24, Issue 5, Sept. 2007.

[9] C. B. Barneto, et al., "Full-Duplex OFDM Radar with LTE and 5G NR Waveforms: Challenges, Solutions, and Measurements," IEEE Trans. Microw. Theory Tech., vol. 67, no. 10, pp. 4042-4054, Oct. 2019.

[10] Z. Zhang et al., "Full-Duplex Wireless Communications: Challenges, Solutions, and Future Research Directions," Proc. IEEE, vol. 104, no. 7, pp. 1369-1409, July 2016.

[11] A. C. Cirik et al., "Robust Transceiver Design in Full-Duplex MIMO Cognitive Radios," IEEE Trans. Veh. Tech., vol. 67, no. 2, Feb. 2018.

[12] NR; Base Station (BS) Radio Transmission and Reception Release 15, document TS 38.104 v15.4.0, Tech. Spec. Group Radio Access Network, 3GPP, Dec. 2018.

[13] H. Luo et al., "Wide-Band Active Analog Self-Interference Cancellation for 5G and Beyond Full-Duplex Systems," 54th Asilomar Conf. on Signals, Systems and Computers," California, Nov. 2020.

[14] A. Celik et al., "Hybrid Energy Harvesting-Based Cooperative Spectrum Sensing and Access in Heterogeneous Cognitive Radio Networks," IEEE Trans. Cogn. Commun. Netw., vol. 3, no. 1, pp. 37-48, Mar. 2017.

[15] C. Galiotto et al., "Unlocking the Deployment of Spectrum Sharing with a Policy Enforcement Framework," IEEE Access, vol. 6, pp. 1179311803, 2018.

\section{BIOGRAPHIES}

Sudip Biswas is an Assistant Professor at ECE Department, IIIT Guwahati, India. He leads research in optimization and signal processing for $5 \mathrm{G} \&$ Beyond Communications. He is currently leading a SERB (DST, Govt. of India) funded project "Signal Processing for Co-existence between Radar and Future Communication Systems" as PI. Earlier, he has worked at IDCOM, University of Edinburgh, UK and Tata Consultancy Services (TCS), India.

Abhijeet Bishnu received his $\mathrm{PhD}$ degree from IIT, Indore, India, in 2019. He is presently a postdoctoral researcher at IDCOM, University of Edinburgh, UK. His research interests include signal processing for wireless communications, cognitive radio, and fullduplex communication.

Faheem A. Khan is a Senior Lecturer at School of Computing and Engineering, University of Huddersfield, UK. He leads research in spectrum sharing for beyond 5G networks, non-orthogonal multiple access and full-duplex communications. He is a Co-Investigator of H2020 ETN and RISE research projects.

Tharmalingam Ratnarajah is a Professor in Digital Communications and Signal Processing at IDCOM, University of Edinburgh, UK. His research interests include signal processing and information-theoretic aspects of beyond $5 \mathrm{G}$ networks, random matrices theory, mmWave communications, full-duplex communications, quantum information theory and statistical and array signal processing. He holds 4 U.S. patens and has published over 400 technical articles. 


\begin{tabular}{|c|c|c|c|c|c|}
\hline $\begin{array}{c}\text { Spectrum } \\
\text { Sharing Approach }\end{array}$ & $\begin{array}{l}\text { Frequency } \\
\text { Band }\end{array}$ & $\begin{array}{l}\text { Sharing } \\
\text { Structure }\end{array}$ & $\begin{array}{l}\text { Management } \\
\text { Blocks }\end{array}$ & Applications & Limitations \\
\hline $\begin{array}{l}\text { Cognitive } \\
\text { Radio }(\mathrm{CR})\end{array}$ & $\begin{array}{c}\text { TV White } \\
\text { Space } \\
470-790 \\
\text { MHz (UK) }\end{array}$ & Interweave & $\begin{array}{c}\text { Spectrum } \\
\text { sensing/Database } \\
\text { assisted }\end{array}$ & $\begin{array}{c}\text { Rural } \\
\text { broadband }\end{array}$ & $\begin{array}{c}\text { No QoS } \\
\text { guarantees for } \\
\text { secondary users }\end{array}$ \\
\hline $\begin{array}{l}\text { Licensed } \\
\text { Shared Access } \\
\text { (LSA) }\end{array}$ & $\begin{array}{l}2.3-2.4 \\
\mathrm{GHz} \\
\text { Europe }\end{array}$ & $\begin{array}{c}\text { Two-tier } \\
\text { sharing } \\
\text { (incumbent and } \\
\text { licensee) }\end{array}$ & $\begin{array}{c}\text { LSA } \\
\text { Repository, LSA } \\
\text { Controller }\end{array}$ & $\begin{array}{r}\text { Mobile } \\
\text { Broadband }\end{array}$ & $\begin{array}{c}\text { Target } \\
\text { stakeholders } \\
\text { (MNOs) still } \\
\text { focused on } \\
\text { dedicated licensed } \\
\text { spectrum }\end{array}$ \\
\hline $\begin{array}{c}\text { Citizens } \\
\text { Broadband Radio } \\
\text { Service- Spectrum } \\
\text { Access System } \\
\text { (CBRS-SAS) }\end{array}$ & $\begin{array}{c}3.55-3.7 \\
\text { GHz USA }\end{array}$ & $\begin{array}{c}\text { Three-tier } \\
\text { sharing model } \\
\text { involving IA, } \\
\text { PAL and GAA } \\
\text { users }\end{array}$ & $\begin{array}{c}\text { Spectrum } \\
\text { Access System }\end{array}$ & $\begin{array}{r}\text { Mobile } \\
\text { Broadband }\end{array}$ & $\begin{array}{c}\text { No QoS } \\
\text { guarantees for GAA } \\
\text { users }\end{array}$ \\
\hline
\end{tabular}

Table 1: Comparison of current dynamic spectrum sharing approaches 


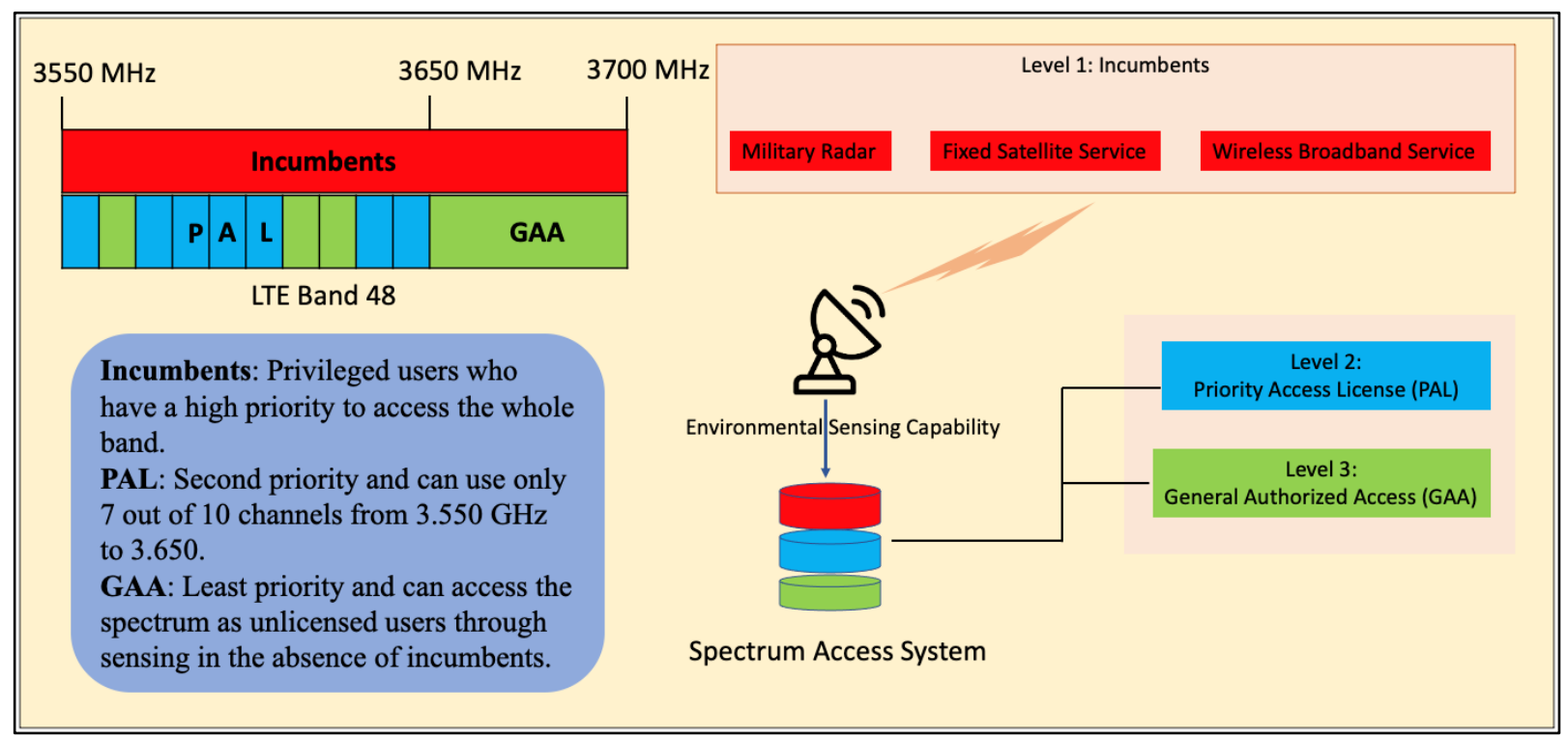

Figure 1: Three tier CBRS based on SAS as approved by FCC. 


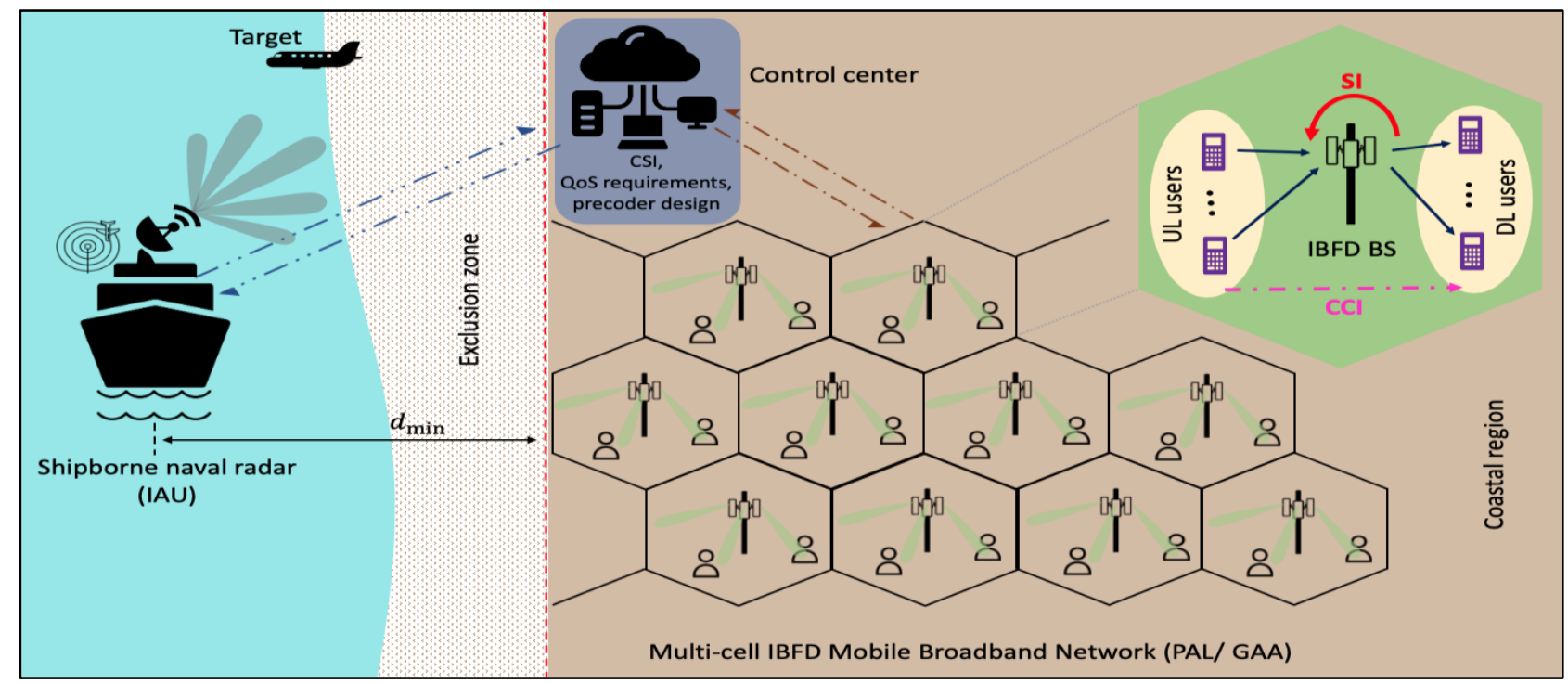

Figure 2: IBFD CBRS Network Architecture 


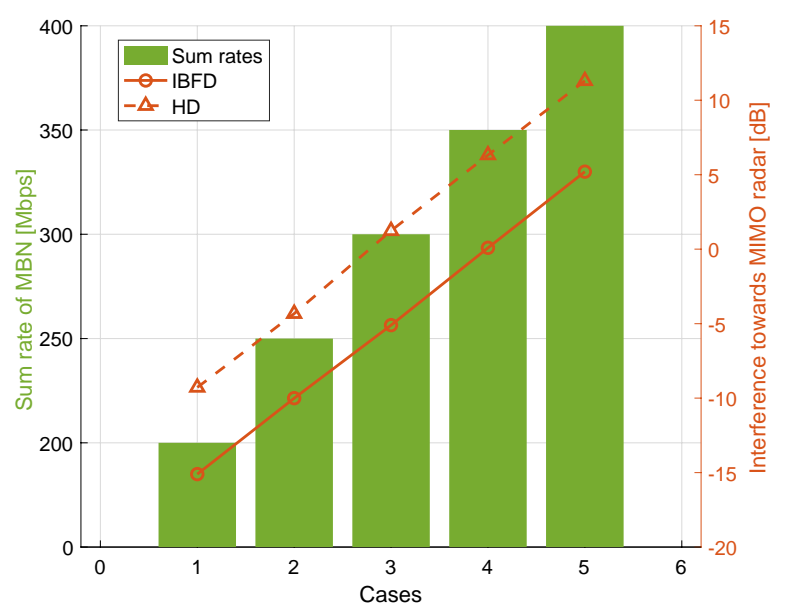

Figure 3: Interference from MBN towards IU for different QoS 


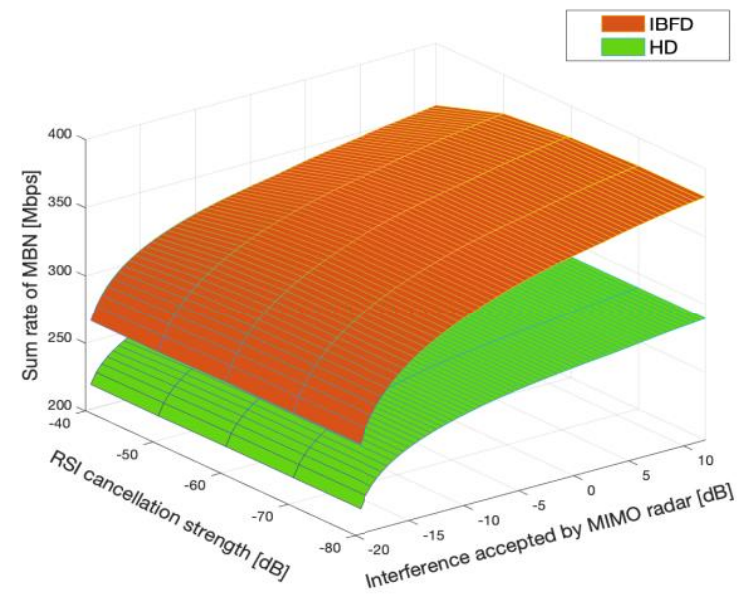

Figure 4: Sum rate of the MBN vs RSI cancellation strength vs Interference accepted by MIMO radar 


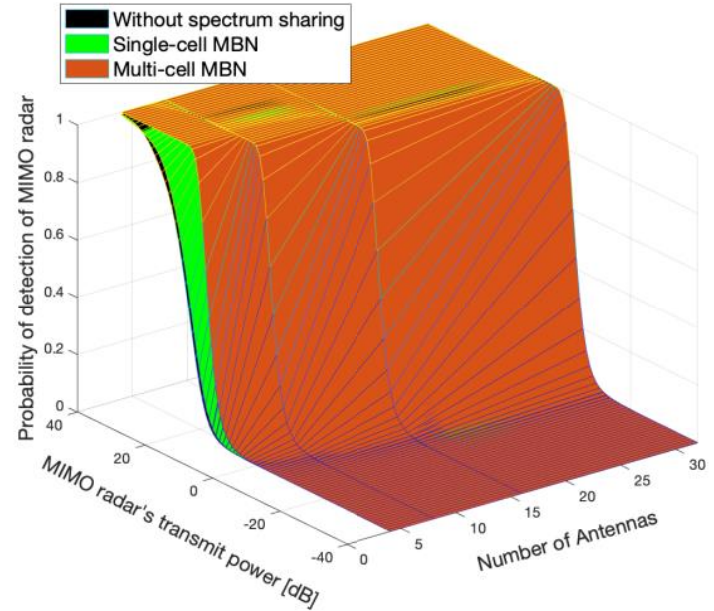

Figure 5: Probability of detection of the MIMO radar 